\title{
Clinical signs and symptoms in a joint model of four disease activity parameters in juvenile dermatomyositis: a prospective, longitudinal, multicenter cohort study
}

E. H. Pieter van Dijkhuizen ${ }^{1,2,3}$, Maria De Iorio ${ }^{4}$, Lucy R. Wedderburn ${ }^{3,5,6 \dagger}$, Claire T. Deakin ${ }^{3 * \dagger}$ and on behalf of the JDRG

\begin{abstract}
Background: It is currently impossible to predict the prognosis of patients with juvenile dermatomyositis (JDM). The aim of this study was to find clinical features most strongly associated with outcome variables in JDM as a first step towards tailor-made treatment.

Methods: In a large, prospectively followed, multicenter cohort study of 340 patients with JDM, each contributing multiple visits, a Bayesian model of disease activity was developed, using the four continuous outcome variables creatine kinase (CK), childhood myositis assessment score (CMAS), manual muscle testing of 8 muscle groups (MMT8) and the physician's global assessment of disease activity (PGA). Covariates were clinical signs and symptoms. Correlations among visits of the same patient were resolved by introducing subject-specific random effects.

Results: Myalgia and dysphonia were associated with worse disease activity according to all outcome variables. Periorbital rash, rash on the trunk, rash over large joints, nail fold changes and facial swelling were associated with higher PGA. Notably, periorbital rash was also associated with higher CK and lower CMAS and nail fold changes with lower CMAS. Contractures were associated with lower CMAS and MMT8 and higher PGA. Patients with higher CMAS exhibited a higher MMT8 as well. PGA had the highest probability among the four outcome variables of being abnormal even if the other three outcome variables were normal.

Conclusions: The signs and symptoms associated with disease activity could be used to stratify patients and adapt treatment plans to disease activity. The correlation between CMAS and MMT8 and the unique information captured by PGA implied that PGA should be maintained as an outcome variable, whereas CMAS and MMT8 might be simplified.
\end{abstract}

Keywords: Juvenile dermatomyositis, Disease activity, Bayesian model, Longitudinal data, Clinical associations

\section{Background}

The childhood inflammatory idiopathic myopathies (IIM) are a group of heterogeneous disorders, characterized by chronic skeletal muscle inflammation. Of these, juvenile dermatomyositis (JDM) is the most common, though still a rare disease with an incidence of about 1.9-4.1 per 1,000,000 children [1]. Its hallmark is muscle

\footnotetext{
* Correspondence: c.deakin@ucl.ac.uk

† Lucy R. Wedderburn and Claire T. Deakin contributed equally to this work. ${ }^{3}$ Paediatric Rheumatology, University College London GOS Institute of Child Health, 30 Guilford Street, London WC1N 1EH, UK

Full list of author information is available at the end of the article
}

inflammation characterized by proximal muscle weakness, in concert with skin involvement presenting itself typically as Gottron's papules, heliotrope rash, malar rash or erythema overlying the extensor surfaces of the joints [2]. JDM potentially involves other internal organ systems as well, most notably the gastrointestinal and the respiratory tracts and patients with major organ involvement have a poor prognosis [2]. JDM is heterogeneous in nature, in terms of disease severity and various patterns of involvement of muscle, skin and internal organ systems. About $24-40 \%$ of patients experience a

(c) The Author(s). 2018 Open Access This article is distributed under the terms of the Creative Commons Attribution 4.0 International License (http://creativecommons.org/licenses/by/4.0/), which permits unrestricted use, distribution, and 
monocyclic course, whereas $50-60 \%$ have chronic disease activity [2]. The mortality rate is around $2-3 \%$ [2].

Given the severity and burden of the disease and possible long-term complications, adequate treatment is of utmost importance [2,3]. A recent trial has shown that the combination of prednisone and methotrexate has the best potential to induce disease remission in new-onset JDM [4]. Ideally, treatment is tailored to the patient, in such a way that patients with high disease activity, at risk of developing serious sequelae of the disease, receive early and aggressive treatment, whereas those with less severe forms of the disease receive milder therapy. Previous studies have revealed some clinical factors associated with a worse prognosis $[1,5,6]$. However, in these reports disease activity was either taken dichotomously at a single point in time or was analyzed as time to remission.

The aim of the current study was to find clinical signs and symptoms associated with higher disease activity as measured by four widely used continuous outcome variables, assessed longitudinally in a large, multicenter cohort of patients with JDM. Such associations with high disease activity could be used in follow up studies to predict disease outcome in patients with JDM as a first step towards tailor-made treatment.

\section{Methods}

Patients were retrieved from the ongoing UK Juvenile dermatomyositis cohort and biomarker study (JDCBS), which started recruitment of patients with JDM across the UK in 2000 [7]. Patients were enrolled at diagnosis or shortly thereafter and followed up approximately every 3 months for 2 years and subsequently at least annually. At each visit, data were collected on signs and symptoms of the disease, such as skin manifestations (e.g., periorbital rash, periungual rash, Gottron's papules, nail fold changes, ulceration), muscular involvement (e.g., muscle weakness, dyspnoea, dysphonia, dysphagia) and symptoms of involvement of other organ systems (e.g., arthritis, chest pain, abdominal pain, hematuria, melena; see Additional file 1 for full list). Blood was drawn for routine laboratory testing. Furthermore, data were collected on treatment and disease activity according to four widely used continuous outcome variables, i.e., creatine kinase (CK), childhood myositis assessment scale (CMAS) [8], manual muscle testing of 8 muscle groups (MMT8) and the physician's global assessment of disease activity (PGA). These outcomes were selected because they have been validated as a set of parameters able to classify patients with JDM as active or inactive [9]. Furthermore, they are widely used and readily available in routine daily care. However, rather than applying previously published criteria for inactive disease and dichotomizing patients as active or inactive, we modeled the four parameters in a continuous way, thus taking full advantage of the information they contain.

Ethical approval was obtained by the multicenter ethical review board covering all participating institutions. All participants provided written informed consent, or age-appropriate assent with parental consent. The study was performed according to the declaration of Helsinki and good clinical practice guidelines.

The UK JDM study enrolled patients with suspected or definite myositis with symptoms starting before the 16th birthday. At the time of analysis, data on $469 \mathrm{pa}-$ tients from 4122 visits were available. Of these, 413 patients contributing 3881 visits met the inclusion criteria of having probable or definite JDM according to the Bohan and Peter criteria [10, 11].

\section{Statistical analysis}

The data were analyzed using a Bayesian approach. Details of the analysis have been described elsewhere [12]. Briefly, a mixed effect regression model was fitted, by specifying a joint model for the four clinical outcome variables. These outcome parameters were modeled as continuous variables. This approach allowed us to account for the correlation among disease activity measures and, therefore, better exploit the information contained in the variables, compared to an analysis that considers the four outcomes as independent and treats the responses as dichotomous. In the analysis, $\mathrm{CK}$ values were log-transformed so that the distribution of CK was closer to normal. On the other hand, CMAS, MMT8 and PGA were square root transformed, as they are non-negative variables potentially assuming value zero. More interestingly, these three variables showed an excess of the best possible clinical value for that parameter for visits of patients in disease remission and a long tail towards the pathological end of the scale. These distributional characteristics must be accounted for in the analysis to avoid bias in the estimates. As such we modeled CMAS, MMT8 and PGA using an approach similar to hurdle models [13]. This approach allowed the model to estimate many more visits of patients to be in disease remission than a standard linear regression would do, thus mirroring the observed distribution of the outcome parameters.

To make maximal use of the information contained in the dataset, all visits of all patients were analyzed simultaneously. Temporal correlation between multiple visits of the same patient was accounted for using a subject-specific random intercept and, in the case of CK level, a subject-specific random slope for the time since diagnosis, in a way similar to a mixed model in a repeated measurements analysis [14]. Furthermore, due to correlations between the four outcome parameters, the four subject-specific random intercepts (one for each outcome variable) were modeled by specifying a 
multivariate normal distribution as random effect distribution. The covariance matrix of this distribution was estimated from the data [15]. This allowed the assessment of the correlation among the outcome parameters (i.e., the correlation that measures whether one outcome parameter tended to increase if another parameter increased as well within the same individual over time). Missing values in the covariates were imputed in the Bayesian model, by specifying an appropriate model for them. We did not impute missing values for the history variables, since this would amount to imputing a participant's recollection, the accuracy of which is doubtful. Visits with any unobserved history variables were therefore excluded from the analysis.

The aim of the model was to find all clinical features associated with disease activity. Therefore, all clinical signs and symptoms and treatment variables, and the time elapsed since diagnosis were eligible to be included as independent variables in the model. Variable pre-selection was performed by selecting $50 \%$ of the variables using univariate linear mixed models. All treatment covariates and the time elapsed since diagnosis were included in the model, regardless of their performance in the univariate analysis. Bayesian variable selection on the pre-selected covariates was performed [16]. This approach yields the sparsest set of predictors that is still able to estimate the outcome parameters accurately. A predictor variable would either be included for all four outcomes or excluded.

The goodness of fit of the model was assessed visually by checking the fit of the model for patients with more than 10 visits, by looking at 95\% Bayesian credible intervals $(\mathrm{CI})$ for the disease trajectory over time. Furthermore, the ability of the model to predict values above or below the validated cutoff points $(\mathrm{CK} \leq 150$, CMAS $\geq$ 48, MMT $8 \geq 78$ and PGA $\leq 0.2$ ) [9], was assessed by calculating the scaled Brier score. This score has a range from 0 to $100 \%$ and has an interpretation similar to Pearson's $R^{2}$ statistic [17]. The out-of-sample prediction ability of the model was tested by randomly selecting five fully observed patients and leaving them out while fitting the model. The predicted values for the four outcomes over time were then compared to the observed values. Statistical analysis was performed in R 3.2.2 ( $\mathrm{R}$ foundation for statistical computing, Vienna, Austria) and JAGS, using the package rjags [18].

\section{Results}

Due to exclusion of visits with missing data on history variables, 340 of 413 patients were included in the analysis. Of these, the majority (69.4\%) was female, the median age at diagnosis was 7.4 years (1st-3rd quartile 4.5-10.5) and disease duration since the onset of the first symptoms was short (median 0.3 years, 1st-3rd quartile $0.2-0.6)$. Patients contributing more visits to the study were more likely to have at least one visit without missing values in the history variables and were therefore more likely to be included. Excluded patients due to missing data in the history variables appeared to have shorter duration of follow up in the study, longer period of time after diagnosis before enrollment and less active disease (Table 1). The proportion of missing data in the analyzed data set was $7.3 \%$.

The goodness of fit of the model was good as evidenced by a plot of the observed values versus the predicted values and 95\% Bayesian CI (Fig. 1). Over all visits and outcome parameters, only $1.8 \%$ of observed values were outside of the CIs. The scaled Brier score was $49 \%, 42 \%, 63 \%$ and $80 \%$ of the maximally obtainable Brier score for CK, CMAS, MMT8 and PGA, respectively. The visual check of the out-of-sample predictions showed accurate predictions of the four outcomes. Only in two cases involving CMAS was the predicted value above the cutoff point for inactive disease (48 points), whereas the observed value was below, but in both these cases the observed value was close to the cutoff point (46 points and 47 points, respectively). The precision of the predictions was modest as evidenced by wide prediction CIs, owing to uncertainties in predictions, parameter estimation and missing value imputation.

Estimates of regression coefficients of the continuous component of the model showed that CMAS, MMT8 and PGA tended to normalize over time, whereas hardly any influence of time on CK was noted (Fig. 2). Many muscular symptoms, such as myalgia and dysphonia were associated with higher disease activity. Cutaneous symptoms such as periorbital rash, rash on the trunk, rash over large joints, nail fold changes and facial

Table 1 Baseline table

\begin{tabular}{lll}
\hline Parameter & $\begin{array}{l}\text { Included } \\
N=340\end{array}$ & $\begin{array}{l}\text { Excluded } \\
N=73\end{array}$ \\
\hline Female, $n$ (\%) & $236(69.4)$ & $54(74.0)$ \\
Age at diagnosis, years & $7.4(4.5,10.5)$ & $7.3(4.1,11.1)$ \\
Disease duration at diagnosis, years & $0.3(0.2,0.6)$ & $0.3(0.2,1.0)$ \\
Time after diagnosis at enrollment, years & $0.2(0.1,1.1)$ & $2.3(0.4,5.4)$ \\
Duration of follow up, years & $4.1(1.6,7.1)$ & $1.2(0.1,2.6)$ \\
Disease activity at enrollment: & & \\
CK, U/L & $103(64,440)$ & $98(45,256)$ \\
CMAS, points & $41(21,50)$ & $46(37,52)$ \\
MMT8, points & $65(45,80)$ & $80(64,80)$ \\
PGA, cm & $3(1.3,6.0)$ & $2.3(0.5,4.0)$ \\
\hline
\end{tabular}

Values are the median (1st quartile, 3rd quartile), except where indicated otherwise

Abbreviations: $C K$ creatine kinase, $\mathrm{cm}$ centimeter, CMAS childhood myositis assessment scale, MMT8 manual muscle testing of 8 muscle groups, $P G A$ physician's global assessment of disease activity, $U / L$ units per liter 


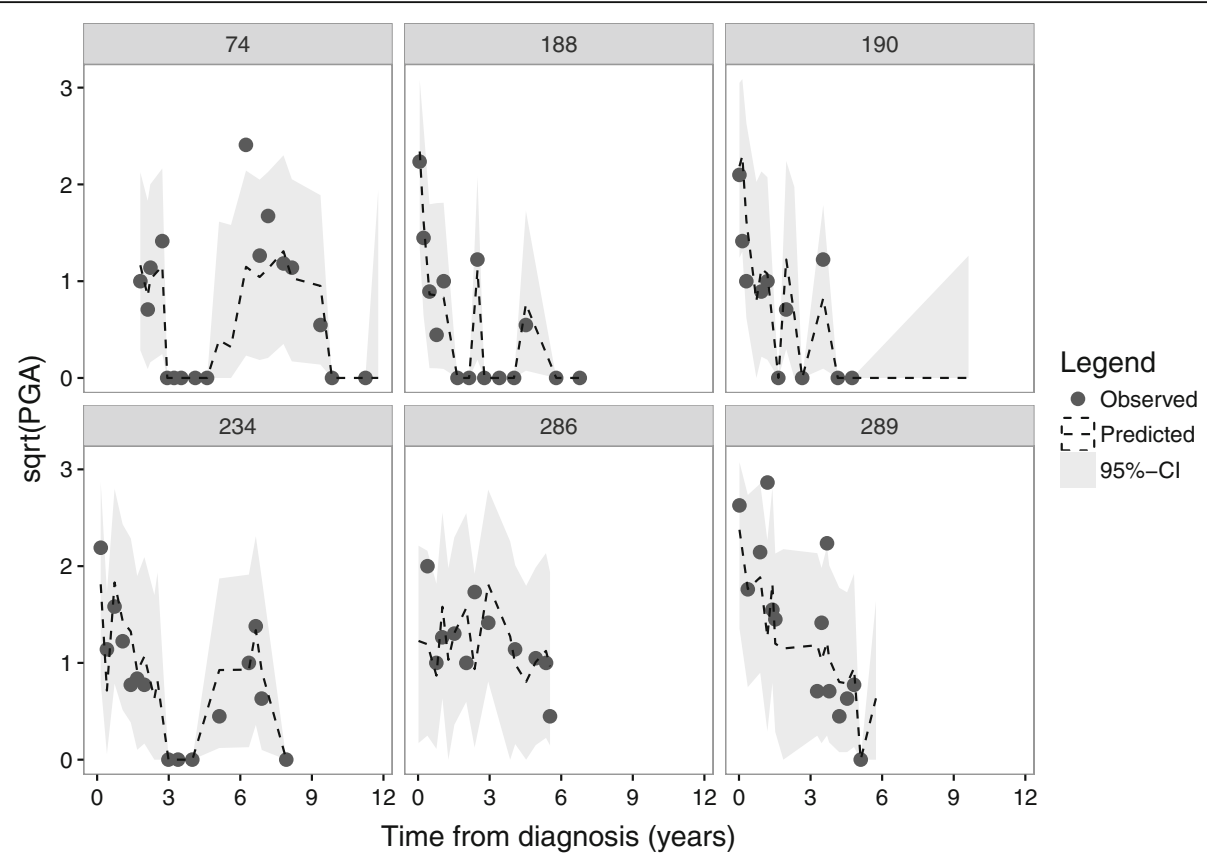

Fig. 1 Goodness of fit of the physician's global assessment of disease activity. Observed values of the (square root transformed) parameter (dark gray dots) for six randomly selected individuals were plotted against the predicted values by the model (dashed line) and the $95 \% \mathrm{Cl}$ (light gray area), showing that the predicted values corresponded well to observed patterns over time. Goodness of fit of the other outcome parameters was similar (not shown). Abbreviations: Cl, credible interval; sqrt, square root; PGA, Physician's global assessment of disease activity

swelling were associated with higher disease activity and, notably, were in some cases associated with more disease activity according to strictly muscular outcome parameters (e.g., periorbital rash was associated with higher CK values and lower CMAS and nail fold changes was associated with lower CMAS).

The presence of contractures was associated with lower CMAS and MMT8 values and higher PGA, whereas CK was not affected. An association was found between calcinosis and higher CMAS and PGA values. Joint swelling was associated with lower CMAS, lower CK and higher PGA and the presence of hematuria was accompanied by markedly elevated CK levels. Of all signs and symptoms at baseline, only arthritis was associated with increased CMAS and MMT8.

The use of both cyclophosphamide and intravenous (IV) steroids was associated with higher disease activity, though cyclophosphamide was also associated with lower CK levels. Conversely, oral steroids were associated with lower disease activity.

Correlations between the subject-specific random intercepts showed that patients with a higher CMAS tended to have a higher MMT8 as well (Table 2). Weak correlation was found between MMT8 and PGA and CMAS and PGA. All correlations between the subject-specific random intercept for $\mathrm{CK}$ and the other three parameters were low (Table 2).
Of visits during which three outcome variables had normal values, the median (1st, 3rd quartile) probability that the predicted value for PGA remained abnormal (i.e. > $0.2)$ was estimated to be $52 \%(<1,78 \%)$. The median (1st, 3rd quartile) probability was $6 \%(2,20 \%), 1 \%(<1,8 \%)$ and $1 \%(<1,17 \%)$, for CK, CMAS and MMT8 respectively.

\section{Discussion}

This study identified clinical signs and symptoms associated with four outcome parameters taken continuously and longitudinally in a large, multicenter cohort of patients with JDM. This approach not only allowed to estimate the associations between various signs and symptoms and disease activity, but also enabled to assess correlations among the outcome parameters, in order to have a clearer understanding of disease activity by accounting jointly for all the outcome values. The results showed as expected that dysphonia, already known to be a marker of severe disease activity [2], was associated with higher disease activity. Hematuria was also associated with markedly elevated CK levels. Hematuria was measured by urine dipstick, which gives a positive result in the case of hematuria or myoglobinuria. In the former, hematuria is an indication of severe systemic (i.e., renal) disease. In the latter, myoglobinuria is due to severe muscular involvement, leading to rhabdomyolysis.

Cutaneous symptoms were associated with PGA, the only parameter taking account of cutaneous disease 


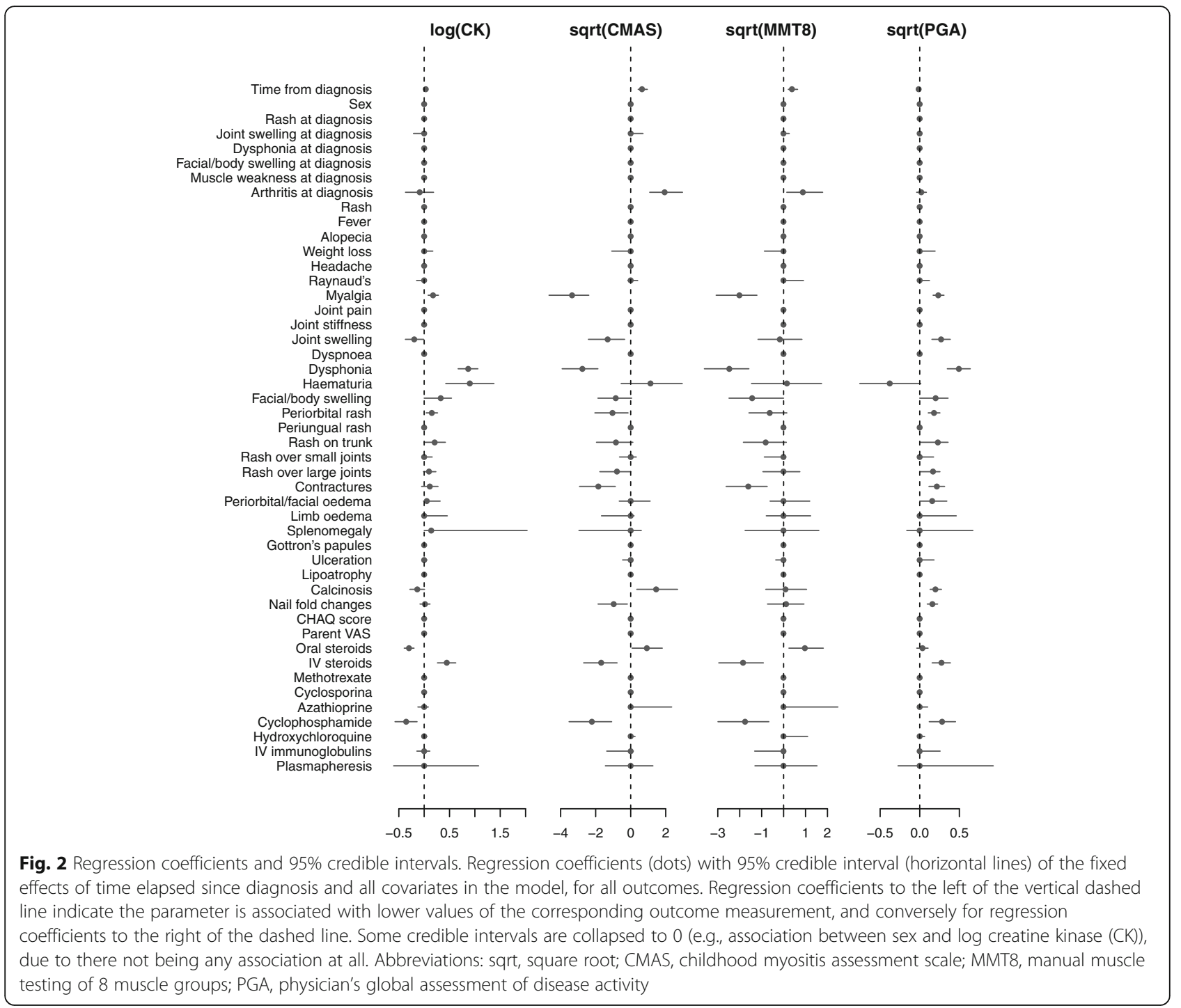

Table 2 Estimated correlations with 95\% credible intervals of subject random intercepts for the four outcomes CK, CMAS, MMT8 and PGA

\begin{tabular}{lll}
\hline Comparison & $\hat{\rho}$ & $95 \% \mathrm{Cl}$ \\
\hline CK vs. CMAS & 0.07 & $(-0.07,0.23)$ \\
CK vs. MMT8 & -0.001 & $(-0.16,0.15)$ \\
CK vs. PGA & 0.0007 & $(-0.15,0.16)$ \\
CMAS vs. MMT8 & 0.54 & $(0.42,0.65)$ \\
CMAS vs. PGA & 0.17 & $(0.01,0.32)$ \\
MMT8 vs. PGA & 0.23 & $(0.08,0.32)$ \\
\hline
\end{tabular}

Abbreviations: $\mathrm{Cl}$ credible interval, $C K$ creatine kinase, $C M A S$ childhood myositis assessment scale, MMT8 manual muscle testing of 8 muscle groups, PGA physician's global assessment activity, but, interestingly, periorbital rash and nail fold changes were also associated with lower CMAS values and higher CK values, implying that cutaneous and muscular disease were correlated. It could therefore be hypothesized that the return of cutaneous symptoms in a patient in disease remission signals an imminent muscular relapse. This finding lends support to the expert-based opinion that ongoing skin disease reflects ongoing systemic disease activity [19]. Interestingly, Gottron's papules, pathognomonic of JDM, were not associated with any outcome parameter.

The association between contractures and lower CMAS and MMT8 values might be due to difficulty in applying these instruments in the presence of contractures [20], especially given the absence of association with CK. Likewise, the association between calcinosis and higher CMAS levels might indicate that this phenomenon occurred in a late stage of disease and 
persisted in disease remission. Yet, the results showed that physicians gave higher PGA scores to patients with calcinosis.

Arthritis at diagnosis was associated with increased CMAS and MMT8, potentially due to a subset of patients with an overlap between JDM and juvenile idiopathic arthritis (JIA) with less severe muscular inflammation [2]. Joint swelling at the visit was associated with a higher PGA and lower CMAS, possibly indicating difficulty in executing the tasks of the CMAS in the presence of arthritis.

The substantial correlation between the subject-specific random intercepts for CMAS and MMT8 indicated that patients with a higher CMAS tended to have a higher MMT8, as reported previously [20]. This implied that information captured by CMAS was also partially captured by MMT8 and vice versa. Conversely, in many visits where CK, CMAS and MMT8 were normal, the model estimated PGA to be abnormal, implying that PGA captures aspects of disease that are not measured by the other outcome parameters. This was consistent with the observation that PGA was the only outcome parameter taking account of cutaneous involvement and involvement of other organs [21, 22]. Similar estimates for the other outcome parameters in our model were much lower, indicating that these variables captured information already conveyed by the other outcome measures. In conclusion, our observations supported the previously made proposal to increase the weight of PGA in the evaluation of JDM disease activity, to account for cutaneous disease activity [22]. Moreover, our results suggested that the three muscular disease activity measures, mainly CMAS and MMT8, could be shortened or even summarized into a single instrument, thus saving precious time during busy clinics, whilst retaining the same level of information.

The results of our study were in line with results obtained previously $[1,5,6]$. In a large cohort of 490 patients with JDM, analyzing the dichotomized outcome parameter at one time point, a mean of 7.7 years after diagnosis, there was association between CMAS and dysphagia and dysphonia [1]. This study also found an increased probability of having a CMAS score of 52 points in patients with cutaneous symptoms at onset, whereas in our study cutaneous manifestations (though not at onset) were associated with a lower CMAS score [1]. In a Canadian cohort of 84 patients, the persistence of skin rash, especially Gottron's papules, 3 months after diagnosis was associated with a longer time to remission [5]. However, given that disease remission was defined in this study as absence of skin rash (including Gottron's papules), myositis and arthritis, this finding might be tautological [5]. Gottron's papules were not associated with any of the four outcome parameters in our study. In a retrospective study of 61 patients with JDM, a lower skin disease activity score (DAS) at baseline was also associated with a monocyclic disease course [6].

A limitation to the current study is the lack of auto-antibody and muscle biopsy data, and indeed any other biomarkers in our data set. The antibody patterns in the childhood IIM have been studied extensively and were found to determine different subsets of the disease, characterized among others by the distribution of skin rash, contractures, dysphonia, dysphagia, and the outcome of the disease [23, 24]. It would be interesting to know if these different subsets entail varying prognoses. Furthermore, prediction of outcome could be aided by muscle biopsy findings $[25,26]$ and yet other biomarkers may be found among blood plasma cytokines, chemokines and other inflammation-related compounds.

The goal of our study was to find clinical signs and symptoms associated with four frequently used disease activity parameters $[9,22]$. As a consequence, no other outcome parameters, mainly cutaneous disease activity measurements, were considered. Future work may address this limitation; however, this would entail cutaneous symptoms that form part of the outcome measurement no longer being available as predictors. Likewise, our study did not contain patient-reported outcomes, nor other important outcomes, such as physical function, damage or health-related quality of life.

Hardly any features at baseline were associated with disease activity at follow up. This was most probably because the model included also signs and symptoms during follow up, the associations of which overwhelmed associations with baseline variables. Future work may investigate the predictive ability of these signs and symptoms in the assessment of disease activity. Attention should also be paid to the predictive ability of CK, CMAS, MMT8 and PGA at baseline and efforts should be undertaken to find out what variables are key to predicting disease outcome in JDM in order to identify a parsimonious set of data that should be collected at all clinical visits.

Finally, the exclusion of visits with missing history variables, led to the exclusion of 73 patients who appeared to have lower disease activity. This was probably due to the fact they were enrolled in the study at a later time point after diagnosis. Moreover, their follow up was shorter than in patients who were included in the analysis (Table 1). Therefore, the exclusion of this biased subset of patients was not considered problematic.

\section{Conclusions}

The associations between clinical signs and symptoms and four continuous disease activity measurements in this large, multicenter cohort of patients with JDM, who were followed longitudinally, open up possibilities to personalize treatment plans in JDM, by offering more 
aggressive treatment to patients with signs and symptoms associated with higher disease activity. Follow up studies may attempt to predict future disease activity using the clinical signs and symptoms that were associated with higher disease activity found in the current study. Furthermore, the associations highlight interesting patterns, such as the association between skin disease and muscular outcome measures. Finally, the correlations between the four outcome measurements provide insight into unique information captured by each parameter and might be helpful in the determination of a parsimonious set of outcome parameters in JDM, for example by summarizing the CMAS and MMT8 in a single instrument and increasing the importance of PGA in the evaluation of disease remission.

\section{Additional file}

Additional file 1: List of available covariates. (DOCX $14 \mathrm{~kb}$ )

\section{Abbreviations \\ Cl: Credible interval; CK: Creatine kinase; CMAS: Childhood myositis assessment scale; DAS: Disease activity scale; IIM: Idiopathic inflammatory myopathies; JDM: Juvenile dermatomyositis; JIA: Juvenile idiopathic arthritis; MMT8: Manual muscle testing of 8 muscle groups; PGA: Physician's global assessment of disease activity}

\section{Acknowledgements}

The Juvenile Dermatomyositis Research Group would like to thank all of the patients and their families who contributed to the Juvenile dermatomyositis cohort and biomarker study and Repository. We thank all local research coordinators and principal investigators who have made this research possible. The JDRG members were as follows: Dr. Kate Armon, Mr. Joe Ellis-Gage, Ms. Holly Roper, Ms. Vanja Briggs and Ms. Joanna Watts (Norfolk and Norwich University Hospitals), Dr. Liza McCann, Mr. lan Roberts, Dr. Eileen Baildam, Ms. Louise Hanna, Ms. Olivia Lloyd and Susan Wadeson (The Royal Liverpool Children's Hospital, Alder Hey, Liverpool), Dr. Phil Riley and Ms. Ann McGovern (Royal Manchester Children's Hospital, Manchester), Dr. Clive

Ryder, Mrs. Janis Scott, Mrs. Beverley Thomas, Professor Taunton Southwood, Dr. Eslam Al-Abadi (Birmingham Children's Hospital, Birmingham), Dr. Sue Wyatt, Mrs. Gillian Jackson, Dr. Tania Amin, Dr. Mark Wood, Dr. Tania Amin, Dr. Vanessa VanRooyen and Ms. Deborah Burton (Leeds General Infirmary, Leeds), Dr. Joyce Davidson, Dr. Janet Gardner-Medwin, Dr. Neil Martin, Ms. Sue Ferguson, Ms. Liz Waxman and Mr. Michael Browne (The Royal Hospital for Sick Children, Yorkhill, Glasgow), Dr. Mark Friswell, Professor Helen Foster, Mrs. Alison Swift, Dr. Sharmila Jandial, Ms. Vicky Stevenson, Ms. Debbie Wade, Dr. Ethan Sen, Dr. Eve Smith, Ms. Lisa Qiao, Mr. Stuart Watson and Ms. Claire Duong (Great North Children's Hospital, Newcastle), Dr. Helen Venning, Dr. Rangaraj Satyapal, Mrs. Elizabeth Stretton, Ms. Mary Jordan, Dr. Ellen Mosley, Ms. Anna Frost, Ms. Lindsay Crate, Dr. Kishore Warrier and Stefanie Stafford (Queens Medical Centre, Nottingham), Professor Lucy Wedderburn, Dr. Clarissa Pilkington, Dr. Nathan Hasson, Mrs. Sue Maillard, Ms. Elizabeth Halkon, Ms. Virginia Brown, Ms. Audrey Juggins, Dr. Sally Smith, Mrs. Sian Lunt, Ms. Elli Enayat, Mrs. Hemlata Varsani, Miss Laura Kassoumeri, Miss Laura Beard, Miss Katie Arnold, Mrs. Yvonne Glackin, Ms. Stephanie Simou, Dr. Beverley Almeida, Dr. Kiran Nistala, Dr. Raquel Marques, Dr. Claire Deakin, Ms. Stefanie Dowle, Dr. Charalampia Papadopoulou, Dr. Shireena Yasin, Mrs. Cerise Johnson-Moore, Ms. Emily Robinson (Great Ormond Street Hospital, London), Dr. Kevin Murray (Princess Margaret Hospital, Perth, Western Australia) Dr. John loannou and Ms. Linda Suffield (University College London Hospital, London) Dr. Muthana Al-Obaidi, Ms. Helen Lee, Ms. Sam Leach, Ms. Helen Smith, Dr. Anne-Marie McMahon, Ms. Heather Chisem and Ruth Kingshott (Sheffield's Children's Hospital, Sheffield); Dr. Nick Wilkinson, Ms. Emma Inness, Ms. Eunice Kendall, Mr. David Mayers, Ruth Etherton, Danielle Miller and Dr. Kathryn Bailey (Oxford University Hospitals, Oxford); Dr. Jacqui Clinch, Ms. Natalie Fineman and Ms. Helen Pluess-Hall (Bristol Royal Hospital for Children, Bristol); Ms. Lindsay Vallance (Royal Aberdeen Children's Hospital); Ms. Louise Akeroyd (Bradford Teaching Hospitals); Dr. Alice Leahy, Amy Collier, Rebecca Cutts, Emma Macleod, Dr. Hans De Graaf, Dr. Brian Davidson, Sarah Hartfree, Danny Pratt (University Hospital Southampton).

\section{Funding}

EHPVD received funding from the 7th Framework programme of the EU, SP3-People, support for training and career development for researchers (Marie Curie), Network for Initial Training (ITN), FP7-PEOPLE-2011-ITN, under the Marie Skłodowska-Curie grant agreement No 289903. CTD was supported by a fellowship from The Myositis Association (TMA). Funding for the UK JDM Cohort and Biomarker study has been provided by generous grants from the Wellcome Trust UK (085860), Action Medical Research UK (SP4252), The Myositis Support Group UK, Arthritis Research UK (14518, 20164), The Henry Smith Charity and Great Ormond Street Children's Charity (V1268), The Myositis Association, The Medical Research Council and the National Institute for Health Research (NIHR) Translational Research Collaboration (TRC) Rare Diseases. This research was supported by the NIHR Great Ormond Street Hospital for Children NHS Foundation Trust Biomedical Research Centre (NIHR-GOSH BRC). The JDM Cohort study is adopted onto the NIHR Comprehensive Research Network. The Arthritis Research UK Centre for Adolescent Rheumatology at UCL, UCL Hospital and GOSH is supported by grants from Arthritis Research UK (20164) and Great Ormond Street Children's Charity. This is a summary of independent research funded by the NIHR's Rare Diseases Translational Research Collaboration. The views expressed are those of the authors and not necessarily those of the National Health Service (NHS), the $\mathrm{NIHR}$ or the Department of Health.

\section{Availability of data and materials}

The data that support the findings of this study are available from the JDCBS Steering Committee but some restrictions apply to the availability of these data, which were used under license for the current study, and so are not publicly available. Data are however available from the authors upon reasonable request and with permission of the JDCBS Steering Committee.

\section{Authors' contributions}

EHPVD was involved in data analysis and interpretation and drafted the first version of the manuscript. CTD was involved in data analysis and interpretation and revised the manuscript critically. MDI was involved in data analysis and interpretation and revised the manuscript critically. LRW was involved in study conception and design and interpretation of data and revised the manuscript critically. All authors read and approved the final manuscript.

\section{Ethics approval and consent to participate}

Ethical approval was obtained by the multicenter ethical review board covering all participating institutions. All participants provided written informed consent, or age-appropriate assent with parental consent.

\section{Consent for publication}

Not applicable.

\section{Competing interests}

The authors declare that they have no competing interests.

\section{Publisher's Note}

Springer Nature remains neutral with regard to jurisdictional claims in published maps and institutional affiliations.

\section{Author details}

${ }^{1}$ Pediatric Rheumatology, University Medical Center Utrecht Wilhelmina Children's Hospital, Utrecht, The Netherlands. Pediatric Rheumatology, IRCCS G. Gaslini, Genoa, Italy. ${ }^{3}$ Paediatric Rheumatology, University College London GOS Institute of Child Health, 30 Guilford Street, London WC1N 1EH, UK. ${ }^{4}$ Department of Statistical Science, University College London, London, UK. ${ }^{5}$ Great Ormond Street Hospital for Children, London, UK. ${ }^{6} \mathrm{NIHR}$ GOSH Biomedical Research Centre (BRC), London, UK. 
Received: 16 February 2018 Accepted: 25 July 2018

Published online: 15 August 2018

\section{References}

1. Ravelli A, Trail L, Ferrari C, Ruperto N, Pistorio A, Pilkington C, et al. Longterm outcome and prognostic factors of juvenile dermatomyositis: a multinational, multicenter study of 490 patients. Arthritis Care Res (Hoboken). 2010;62(1):63-72.

2. Rider LG, Katz JD, Jones OY. Developments in the classification and treatment of the juvenile idiopathic inflammatory myopathies. Rheum Dis Clin N Am. 2013:39(4):877-904

3. Kim S, El-Hallak M, Dedeoglu F, Zurakowski D, Fuhlbrigge RC, Sundel RP. Complete and sustained remission of juvenile dermatomyositis resulting from aggressive treatment. Arthritis Rheum. 2009;60(6):1825-30.

4. Ruperto N, Pistorio A, Oliveira S, Zulian F, Cuttica R, Ravelli A, et al. Prednisone versus prednisone plus ciclosporin versus prednisone plus methotrexate in new-onset juvenile dermatomyositis: a randomised trial. Lancet. 2016;387(10019):671-8.

5. Stringer E, Singh-Grewal D, Feldman BM. Predicting the course of juvenile dermatomyositis: significance of early clinical and laboratory features. Arthritis Rheum. 2008;58(11):3585-92.

6. Christen-Zaech S, Seshadri R, Sundberg J, Paller AS, Pachman LM. Persistent association of nailfold capillaroscopy changes and skin involvement over thirty-six months with duration of untreated disease in patients with juvenile dermatomyositis. Arthritis Rheum. 2008;58(2):571-6.

7. Martin N, Krol P, Smith S, Murray K, Pilkington CA, Davidson JE, et al. A national registry for juvenile dermatomyositis and other paediatric idiopathic inflammatory myopathies: 10 years' experience; the juvenile dermatomyositis national (UK and Ireland) cohort biomarker study and repository for idiopathic inflammatory myopathies. Rheumatology (Oxford). 2011;50(1):137-45.

8. Lovell DJ, Lindsley CB, Rennebohm RM, Ballinger SH, Bowyer SL, Giannini $\mathrm{EH}$, et al. Development of validated disease activity and damage indices for the juvenile idiopathic inflammatory myopathies. II. The childhood myositis assessment scale (CMAS): a quantitative tool for the evaluation of muscle function. The juvenile dermatomyositis disease activity collaborative study group. Arthritis Rheum. 1999:42(10):2213-9.

9. Lazarevic D, Pistorio A, Palmisani E, Miettunen P, Ravelli A, Pilkington C, et al. The PRINTO criteria for clinically inactive disease in juvenile dermatomyositis. Ann Rheum Dis. 2013;72(5):686-93.

10. Bohan A, Peter JB. Polymyositis and dermatomyositis (second of two parts). N Engl J Med. 1975;292(8):403-7.

11. Bohan A, Peter JB. Polymyositis and dermatomyositis (first of two parts). N Engl J Med. 1975;292(7):344-7.

12. Van Dijkhuizen EHP, Deakin $C T$, Wedderburn LR, De lorio M. Modelling disease activity in juvenile dermatomyositis: a Bayesian approach. Stat Methods Med Res. 2017; https://doi.org/10.1177/0962280217713233.

13. Mullahy J. Specification and testing of some modified count data models. J Econom. 1986;33:341-65.

14. Pinheiro JC, Bates DM. Mixed-effects models in S and S-PLUS. New York: Springer-Verlag; 2000.

15. Li Q, Pan J, Belcher J. Bayesian inference for joint modelling of longitudina continuous, binary and ordinal events. Stat Methods Med Res. 2016;25(6): 2521-40.

16. Kuo L, Mallick B. Variable selection for regression models. Sankhya Ser B. 1998;60:65-81

17. Steyerberg EW, Vickers AJ, Cook NR, Gerds T, Gonen M, Obuchowski N, et al. Assessing the performance of prediction models: a framework for traditional and novel measures. Epidemiology. 2010;21(1):128-38.

18. Plummer M. rjags: Bayesian Graphical Models using MCMC. R package version 4-6. 2016. https://CRAN.R-project.org/package=riags. Cited 17 Mar 2017.

19. Bellutti Enders F, Bader-Meunier B, Baildam E, Constantin T, Dolezalova P, Feldman BM, et al. Consensus-based recommendations for the management of juvenile dermatomyositis. Ann Rheum Dis. 2017;76(2):329-40.

20. Rider LG, Werth VP, Huber AM, Alexanderson H, Rao AP, Ruperto N, et al Measures of adult and juvenile dermatomyositis, polymyositis, and inclusion body myositis: Physician and Patient/Parent Global Activity, Manual Muscle Testing (MMT), Health Assessment Questionnaire (HAQ)/Childhood Health Assessment Questionnaire (C-HAQ), Childhood Myositis Assessment Scale (CMAS), Myositis Disease Activity Assessment Tool (MDAAT), Disease Activity Score (DAS), Short Form 36 (SF-36), Child Health Questionnaire (CHQ), physician global damage, Myositis Damage Index (MDI), Quantitative Muscle Testing (QMT), Myositis Functional Index-2 (FI-2), Myositis Activities Profile (MAP), Inclusion Body Myositis Functional Rating Scale (IBMFRS), Cutaneous Dermatomyositis Disease Area and Severity Index (CDASI), Cutaneous Assessment Tool (CAT), Dermatomyositis Skin Severity Index (DSSI), Skindex, and Dermatology Life Quality Index (DLQI). Arthritis Care Res (Hoboken). 2011;63(Suppl 11):S118-57.

21. Nistala K, Wedderburn LR. Update in juvenile myositis. Curr Opin Rheumatol. 2013;25(6):742-6.

22. Almeida B, Campanilho-Marques R, Arnold K, Pilkington CA, Wedderburn LR, Nistala K. Analysis of published criteria for clinically inactive disease in a large juvenile dermatomyositis cohort shows that skin disease is underestimated. Arthritis Rheumatol. 2015;67(9):2495-502.

23. Rider LG, Shah M, Mamyrova G, Huber AM, Rice MM, Targoff IN, et al. The myositis autoantibody phenotypes of the juvenile idiopathic inflammatory myopathies. Medicine (Baltimore). 2013;92(4):223-43.

24. Shah M, Mamyrova G, Targoff IN, Huber AM, Malley JD, Rice MM, et al. The clinical phenotypes of the juvenile idiopathic inflammatory myopathies. Medicine (Baltimore). 2013;92(1):25-41.

25. Deakin CT, Yasin SA, Simou S, Arnold KA, Tansley SL, Betteridge ZE, et al. Muscle biopsy findings in combination with myositis-specific autoantibodies aid prediction of outcomes in juvenile dermatomyositis. Arthritis Rheumatol. 2016;68(11):2806-16.

26. Miles L, Bove KE, Lovell D, Wargula JC, Bukulmez H, Shao M, et al. Predictability of the clinical course of juvenile dermatomyositis based on initial muscle biopsy: a retrospective study of 72 patients. Arthritis Rheum. 2007;57(7):1183-91.

\section{Ready to submit your research? Choose BMC and benefit from:}

- fast, convenient online submission

- thorough peer review by experienced researchers in your field

- rapid publication on acceptance

- support for research data, including large and complex data types

- gold Open Access which fosters wider collaboration and increased citations

- maximum visibility for your research: over $100 \mathrm{M}$ website views per year

At $\mathrm{BMC}$, research is always in progress.

Learn more biomedcentral.com/submissions 